\title{
FAST TRACKING ICT INFRASTRUCTURE REQUIREMENTS AND DESIGN, BASED ON ENTERPRISE REFERENCE ARCHITECTURE AND MATCHING REFERENCE MODELS
}

\author{
Peter Bernus \\ Griffith University, bernus@cit.gu.edu.au AUSTRALIA \\ Rob Baltrusch \\ Griffith University, rob@cit.gu.edu.au AUSTRALIA \\ Johan Vesterager \\ Technical University of Denmark, jve@ipl.dtu.dk DENMARK \\ Martin Tølle \\ Technical University of Denmark, mat@ipl.dtu.dk DENMARK
}

\begin{abstract}
The Globemen Consortium has developed the virtual enterprise reference architecture and methodology (VERAM), based on GERAM and developed reference models for virtual enterprise management and joint mission delivery. The planned virtual enterprise capability includes the areas of sales and marketing, global engineering, and customer relationship management. The reference models are the basis for the development of ICT infrastructure requirements. These in turn can be used for ICT infrastructure specification (sometimes referred to as 'ICT architecture').Part of the ICT architecture is industry-wide, part of it is industry-specific and a part is specific to the domains of the joint activity that characterises the given Virtual Enterprise Network at hand. The article advocates a step by step approach to building virtual enterprise capability.
\end{abstract}

\section{INTRODUCTION}

The Globemen ${ }^{1}$ Consortium endeavours to develop a Reference Architecture, Methodology, Reference Models and an ICT Architecture to help partners develop the capability to form enterprise networks that can successfully participate in projects to bid for, and execute, one-of-a-kind projects in a virtual enterprise (VE) environment. The main objectives of the partners include better customer relationship management, fast and predictable global engineering activity (including engineering, procurement and construction), as well as after sales service.

\footnotetext{
${ }^{1}$ GLOBEMEN - Global Engineering and Manufacturing in Enterprise Networks, IMS 99004/ IST-1999-60002, http://globemen.vtt.fi
} 
In order to achieve this objective partners need to be in a state of preparedness that includes the acceptance and agreement on certain management and engineering processes, as well as the agreement and installation of an ICT infrastructure that supports such co-operative activity.

While it is true that many companies today are already carrying out such activities, and often with great efficiency, partners in the Globemen consortium agreed that at present a) such co-operative activities are limited to only part of the necessary tasks, and the benefits from an integrated solution are only partially realised; b) the time, cost and risk of jointly undertaken projects is often not predictable enough to sustain profitable business; c) many activities in the supply chain of joint projects could be carried out with greater ease, in better quality and at a lower cost, if the capability of today's ICT were applied in a consistent and integrated manner.

Companies are puzzled by the large number of possible ICT modules and approaches, and it is hard for them to ensure that the investment in such an infrastructure delivers the expected value. For this reason, members decided to use Enterprise Integration / Enterprise Architecture as a discipline to support a coordinated effort to develop a complete and consistent set of requirements, as well as to define an ICT architecture, that is both flexible and able to support all user requirements that follow from their strategic objectives.

The enterprise reference architecture used by the consortium is a combination of particular reference architectures - PERA (Williams, 1994), CIMOSA (Vernadat, 1996), GIM (Doumeingts et al, 1998) and is unified under the umbrella of GERAM (IFIP-IFAC Task Force, 1999). Furthermore, since the reference architecture is to be utilised for the specific purpose of VE capability development, specific details have been added to GERAM, detail that could not be part of a completely generalised architecture (such as GERAM), but is useful in the particular problem at hand. This VE-specific architecture is called VERAM or VERA (Virtual Enterprise Reference Architecture and Methodology) (Zwegers et al, 2001) and (Vesterager et al, 2001). Another article at this conference (Tølle et al, 2001) describes VERA and applies it as a structuring architecture for mapping applicable VE reference models . Throughout this article we use the terminology of GERAM/VERAM.

\section{REFERENCE MODELS TO SUPPORT THE DEVELOPMENT OF ICT ARCHITECTURE REQUIREMENTS}

An 'ICT architecture' is a Reference Model that describes the functions and structure of an ICT system. It is an architecture because it is defined on the level of preliminary (or 'architectural') design, leaving space for various concrete implementation decisions on the detailed design level.

The main aim of the ICT architecture definition is to identify, in addition to the functionality, the interfaces between implementing modules, and to define a certain level of expected interoperability. 
Interoperability in our definition is the ability to exchange information without the need for constant human intervention to ensure correct interpretation. Interoperability between systems may be achieved on different levels.

Low level interoperability only ensures that physically data can be transferred from one system to the other, i.e. sending/receiving various forms of data representation including database records, worksheets, video or picture recordings, drawings, etc.

Medium level interoperability may be defined, for example, if systems are mutually able to access data stored in other systems, e.g. by remote database access.

Higher levels of interoperability may be defined as systems having the ability (without human intervention or help) to interpret data accessed / received from other systems, i.e. data stored in one system are conformant to some commonly agreed-on information schema (semantic integration).

An even higher level of interoperability may be defined as the ability to interact with other systems including the establishment and selection of one of many possible channels of communication (e.g. in case of malfunction, or first time communication), and conduct co-operative dialogues (such as may be achieved by agents, in the sense of co-operative agenthood as defined in the artificial intelligence literature, using agent communication protocols (Barbuceanu and Teigen 1998)

This last level of interoperability is becoming more important as it cannot be expected that our systems will at all times be configured according to a pre-agreed and static architecture.

The aim of the definition of a suitable ICT architecture is to support the said functionality (customer relationship management, global engineering and after sales service) and to achieve competitive advantage through creating the highest possible level of interoperability.

The Globemen architecture and associated reference models divide the concerns between

1) What management transactions are necessary between partner companies on the strategic and tactical levels to form a network of companies,

2) What management activities and transactions need to be performed by the network itself, and

3) What activities are relegated to the tactical and day-to-day operational levels when companies undertake joint action in a project enterprise either for joint bidding, or for delivering one-of-a-kind products or services, or when they perform prolonged joint operation in a co-ordinated supply chain.

There are two types of reference model being developed: 1) Functional Reference Models (activity-, decisional- and process- as well as information-) which are models to establish the functional and information requirements that must be satisfied; 2) ICT Reference Model (ICT architecture) to describe a sufficiently generic composition of systems that can then be implemented in support of the requirements models (Baltrusch, 2000). The Globemen Functional Reference Model is the basis for the development of ICT infrastructure requirements. These in turn can be used for ICT infrastructure specification (sometimes referred to as ICT architecture'). 
Unfortunately, there are so many possible variations of how companies wish to cooperate that there is no unique functional model that could express the requirements of every possible network that partners may wish to create. Therefore, the Globemen Functional Reference Model is produced as a paradigmatic model, which may be tailored or modified by partners to suit their specific case.

As a result, the mapping from the Functional Requirements model to the ICT architecture must include an important step, called orthogonalisation. Orthogonalisation evaluates the functional requirements as derived from user requirements, and defines a function space in which these requirements exist. It is then evaluated what other possible meaningful combinations of functions may exist in this space, and the functional requirements are thus extended to characterise the type of requirements that the ICT infrastructure must satisfy. This extended set is then the basis for ICT architecture specification.

Part of the resulting ICT architecture specification is industry-wide, part of it is industry specific and a part is specific to the domains of the joint activity that characterises the given VE Network at hand. This article advocates a step-by-step approach to building VE capability.

\section{REQUIREMENTS SPECIFICATION: REFERENCE MODELS FOR PARTNERS, NETWORKS AND VIRTUAL PROJECT ENTERPRISES}

\subsection{Functional and Information Requirements Specification of VE Entities}

We use two levels of detail to capture the functional requirements for partners, networks and projects. One level concerns the identification of management (decisional) activities involved in VE creation and operation. This level establishes the context of the more detailed level of specification, which is described in terms of activity and information models. The methodology follows the steps proposed by the GRAI-GIM reference architecture and methodology.

Furthermore, the important division of architectural (preliminary) and detailed design, as well as the conscious attempt to capture all activities (humanimplemented and automated) is the result of applying concepts of the PERA architecture.

This combination of architectures follows the generalised enterprise reference architecture and methodology (GERAM and its VE-specific variation, VERAM).

As known from the GRAI GIM Architecture, one way of capturing both the management and operational requirements of an enterprise is to separately produce a) a management model (using a GRAI-Grid and further representing it in a functional model, such as IDEF0 [note the GRAI methodology advocates the use of GRAI nets for the same purpose]); and b) a functional model of the operations, such as an engineering activity, again using a functional model such as IDEF0 (extended with UML Use Case diagram views), or (if the activity is procedural in nature) a process modelling language, such as IDEF3, CIMOSA, UML sequence diagram, ARIS EPC, or FirstStep. 
Information requirements may be captured in form of IDEF1X, Entity Relationship schemata, Express, or UML Conceptual Schemata. At present the consortium uses a combination of GRAI grids, IDEF0, Use Case diagrams, and UML.

\section{Decisional Models as Top Level Models of Partner, Network and VE Interaction}

While it is true that the VE partners are engaged in a collaborative alliance, a successful VE allows its members to maintain an adequate extent of autonomy (Bernus and Nemes, 1999). Unnecessary detailed control can hinder a partner's ability to draw upon its own resources that would have otherwise optimised resource usage (Ibid). This autonomy should be defined at the network level and disseminated to the work force level as needed. Each partner is sacrificing some autonomy in order to achieve its strategic intent.

The use of decisional models, such as the GRAI-Grid, allows us to represent decisions concerning product and resource management and planning in various enterprise entities these being the Partners, Network and Virtual project enterprises. The GRAI-Grid in Figure 1 (Olegario, 2000) shows a high level abstraction of the main decision flows between these three enterprise entity types.

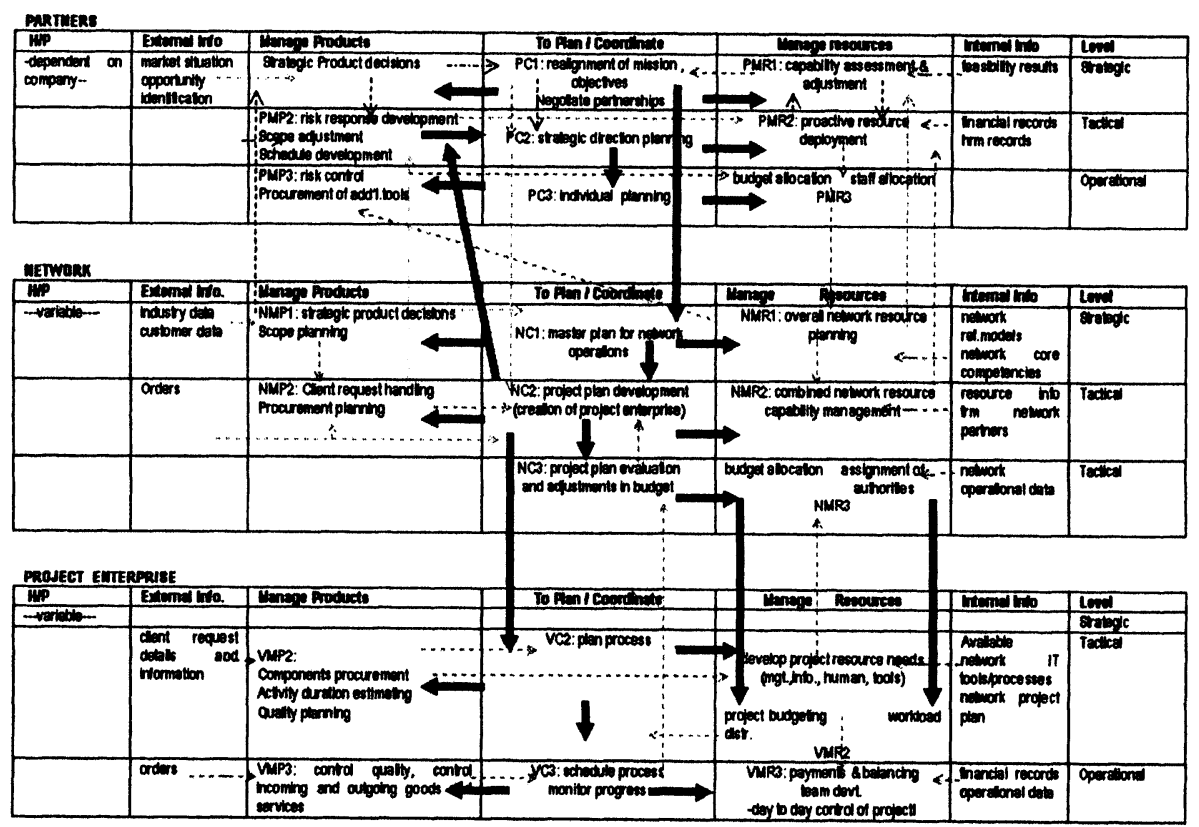

Figure 1 - High Level Abstraction of Main Decision Flows Between Partner, Network and VE (Olegario, 2000)

\section{Activity Models of Partner, Network and VE Interaction}

The step from decisional models to activity models is a non-trivial one. When a decisional model is created, decision centres are linked through decision frameworks, that represent objectives, constraints as well as decision variables (thick arrows in Fig. 1). E.g. Project plan development (as done by the network) determines the objectives and constraints as well as the decision freedom (variables) of project management. However, if we want to represent 'project plan development' 
as an activity model, some parts of the decision framework of project management will contain dynamically changing objectives (thus becoming the control of the project management activity) while some other objectives will be static (as derived from the design of the network), such as network-wide policies regarding the autonomy of project management. Thus it is not possible to automatically transcribe the GRAI grid to a top level activity model; design decisions need to be made regarding what additional requirements project management need to accomplish in the particular type of network, or how it is supposed to accomplish these. Furthermore one needs to decide which of these policies will be taken into account at design time (in the design of the decision activities) and which ones will serve as controls of the decision activity.

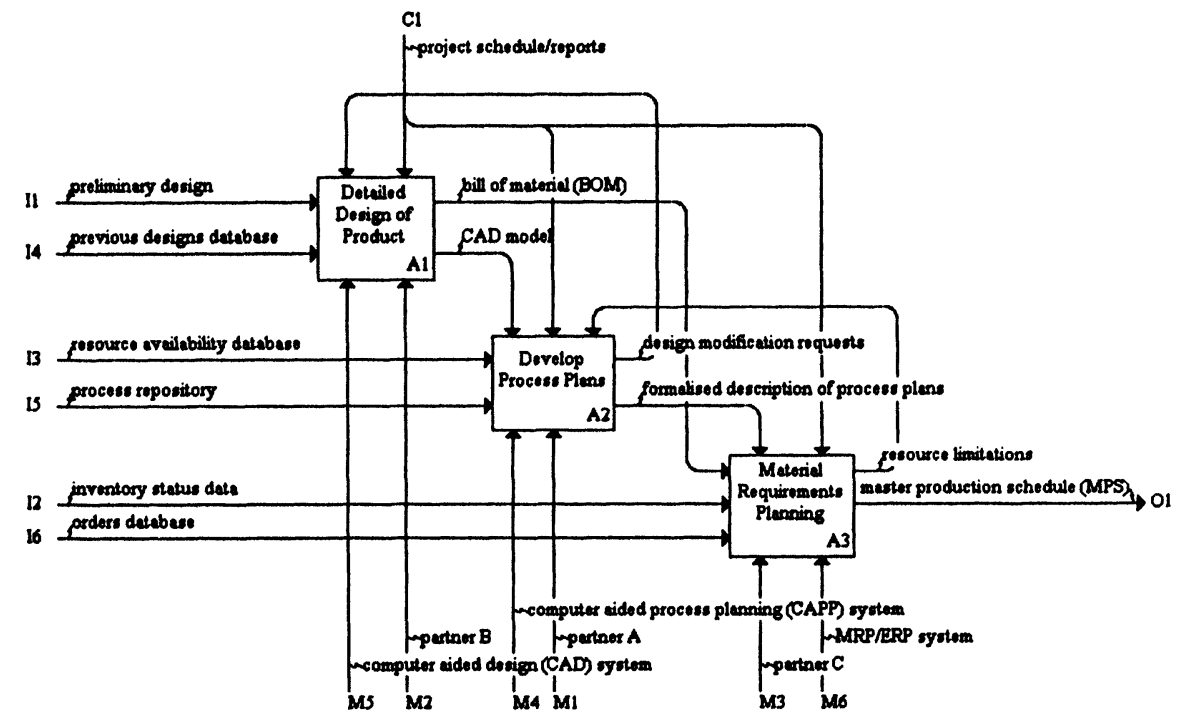

Figure 2 - Distributed Engineering Activity Model and Supporting ICT Application

Modules (Model of an activity in the mission support, i.e. 'Operations')

The second problem in creating activity models for decision centres arises due to the virtual nature of the network and of project enterprises. One way (not chosen) is to describe separately the activities for each enterprise entity (such as network or project) limited to the functions that are performed by the network office or project office, and represent the interfaces of the network or project office to partners as inputs and outputs (or controls) that flow to / from partners or suppliers. The resulting models, however, can easily become crowded and do not show the actual information flow according to the logic of the activity. The other way (followed in the development of our models) is to describe the complete function (whether performed by the network office or project office, or by partners or suppliers). This way of representation shows the activities in the complete process, and thus IDEF0 mechanisms in this model include all stakeholders. (A similar principle can be applied in process modelling as well).

The result is that the interfaces between stakeholders are represented throughout the model, not only on the top level. Decomposition should continue at least to the level, where each activity in the model is performed by only one stakeholder. 
The information requirements then can be identified and expressed using a data modelling language modelling the contents of all those IDEF0 concepts that flow between any two stakeholder.

The low level activities in this model are supported by ICT modules, thus identifying the necessary ICT support for each activity in turn. Note that these ICT supporting modules are only representing the top layers of the ICT architecture (implemented as application modules), since no stakeholder activity uses directly the underlying ICT layers. Thus the ensuing design of ICT architecture must take into account additional information, such as design principles (e.g. regarding the level of interoperability, orthogonalisation, availability of existing technology, etc.)

In order to illustrate the relationship between functional / information requirements and the ICT infrastructure's architectural design, consider the following extract of an activity model in Figure 2, which expresses a function or activity (distributed engineering) on a day to day operational level.

In this construction/production VE example, the particular type of network consists of autonomous partners, each contributing their core competencies for the purposes of the detailed design and implementation of a product.

The activities in the model in Fig.2 are supported by three autonomous companies: partner A, partner B and partner $C$. The network connects them and provides channels for their collaboration. In this example, the partners have full autonomy, and they cooperate with each other through mutually agreed protocols. The interfaces between them are represented by the flow of CAD models and formalised process plans.

Companies A, B and C have agreed upon being partners and working cooperatively on the development of a new product. The profile for the enterprise is: company $\mathbf{B}$ is specialised in design and is responsible for product design using Computer Aided Design (CAD) technology; company A has experts in Computer Aided Process Planning (CAPP), and is chosen as a partner to develop process plans. Company $\mathrm{C}$ is to construct plans and schedules for the production using a Manufacturing Resource Planning (MRP) system.

Here the required ICT application components can be expressed as resources (mechanisms), which support each of the functional activities, and the information required can be expressed as inputs and outputs.

\section{PRELIMINARY DESIGN: MAPPING THE REFERENCE MODELS TO ICT INFRASTRUCTURE REQUIREMENTS}

\subsection{ICT Infrastructure Architectural Design}

Figure 3 below is based on the LISI interoperability maturity model (C4ISR WG, 1998), and shows an example of a Common Operating Environment (COE) or common platform, the goal of which is to establish a commonly defined execution environment for systems. The Shared Data Environment (SHADE) is intended to reach agreement on common data models for systems, in order to standardise data definitions and relationships. 


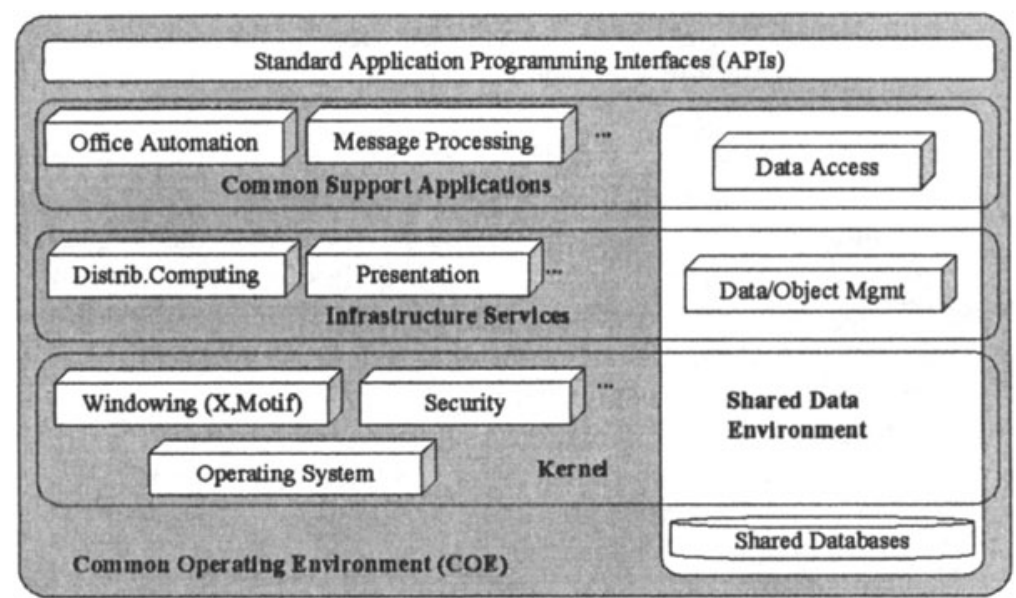

Figure 3 - ICT - Common Operating Environment (COE) Architecture

The COE is based on international (or de-facto) standards therefore it is expected that partners will have no difficulty in adopting these, if they do not already have these services in place.

Figure 4 shows how VE applications can be layered into VE services, such as VE-related communication services, in turn supporting collaboration, and on the highest level, collaboration services.

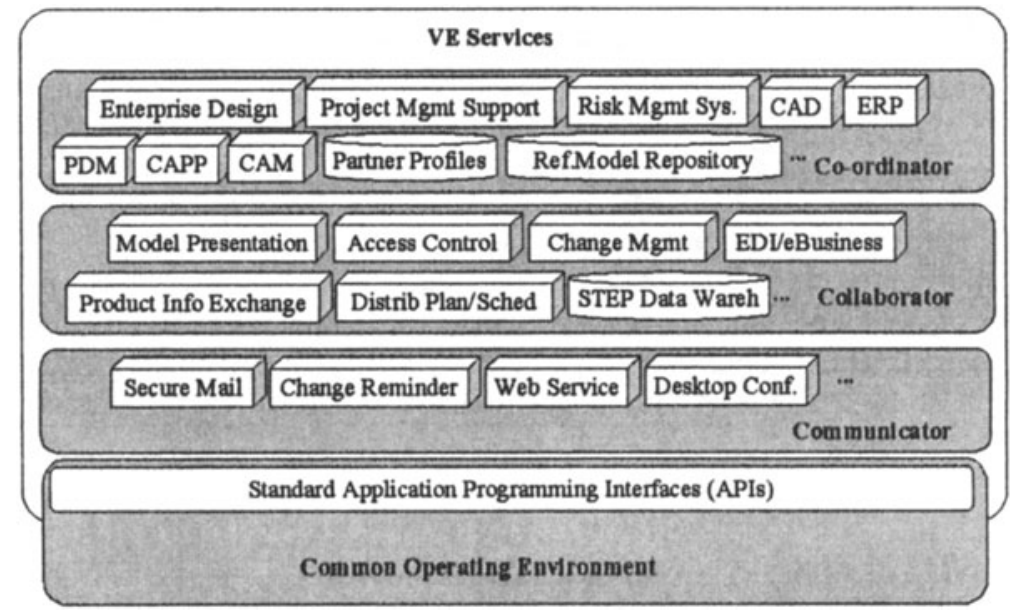

Figure 4 - ICT - Application Layers Architecture (after Zhou et al, 1998 - modified)

It transpires that many existing applications will need extension, with regards to collaborative capabilities, such as systems for planning \& scheduling $v s$. distributed. planning \& scheduling, collaborative design $v s$. stand alone CAD, etc.

In order to design a robust and flexible ICT architecture, it is possible to orthogonalise two aspects: resource and function, such that any given combination of functions can be supported by a certain set of resources. This necessitates the development of suitable abstract data types for resource specification. E.g., instead of separate (specific) editing functions being defined for each type of design object that needs to be collaboratively edited, we need functions that work on any database 
subject to collaboratively performed long design transactions. The decision on the type of the design object must be made independent of these editing functions. Thus the design functions and design object types may be combined in any meaningful manner, whether this combination is stated as a user requirement or not. This ensures that common services are shared across as many domains of business activity as possible. Similar design policies will have to be enforced for the use of collaborative transactions using agent communication protocols.

Figure 5 follows on from our example, and shows the mapping between resource and function, where the resource is conceptually divided into a layered ICT architecture. The better design abstractions we make, the more resource sharing is possible between business functions (domains of activity).

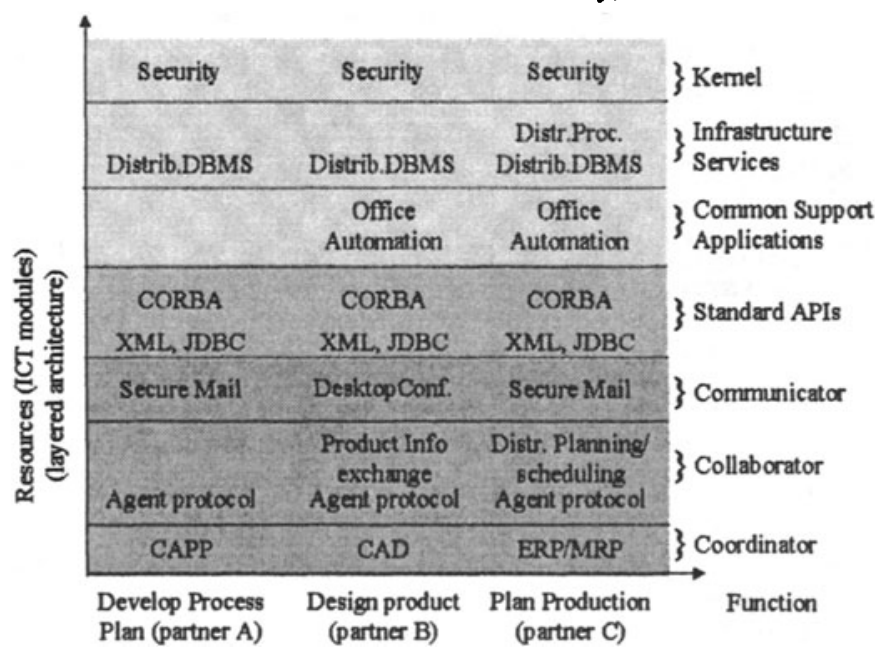

Figure 5 - Mapping ICT Resources and Functions

Since each partner in the network and VE may be responsible for a given subset of activities, only a certain subset of resources will need to be implemented by that partner. Therefore by using orthogonalised resources for the implementation of a specific business function we can isolate a minimal number of ICT components that need to be implemented by specific partners, and through shared design policies interoperability will be ensured. In Fig. 5 Partner A must implement Computer Aided Process Planning (CAPP), XML interfaces and Distributed Computing functionality in the Common Operating Environment. On the other hand, Partner B may already possess the capability to share data using XML as an interface.

It is necessary for the network to pre-agree on a modus operandi, so that it is clear which industry-wide or industry-specific standards are to be used. This requires certain partners to raise their level of maturity regarding the level of interoperability such that they can effectively participate in the network. On the other hand through the application of architectural design policies, as outlined above, the network can select a minimal set of infrastructure modules, improving interoperability and ensuring extendability. 


\section{CONCLUSION}

The Globemen Consortium is designing an ICT architecture to support the highest possible level of interoperability between partners in a VE Network. The approach is based on the GERA/VERAM reference architecture and necessitates the development of two types of reference models: Functional and Structural. The functional reference models include decisional and activity models of the interaction among partners, both in the creation and operation of the network itself, and the creation and operation of virtual project enterprises. The functional models are used to identify the interoperability requirements as well as the necessary set of applications. Further software architecture design is carried out in order to ensure that the quality of the ICT architecture is feasible as well as possessing system properties that make it a long term solution: in both the context of the changing business requirements, such as changes in the type of co-operation among network partners, and in terms of how virtual project enterprises are operated at any one time.

The present state of these models is experimental, and needs validation and further detailing which is being carried out by end user partners in the consortium as well as IT vendor partners, such as ERP vendors.

\section{REFERENCES}

Baltrusch, R., (2000) Infrastructure Requirements for Virtual Manufacturing Enterprises, Honours Dissertation, School of Computing and Information Technology, Brisbane: Griffith University.

Barbuceanu, M. Teigen,R. (1998) System Integration through Agent Coordination. In P.Bernus, K.Mertins and G.Schmidt (Eds) Handbook on Architectures of Information Systems, Berlin: Springer, pp797-826.

Bernus,P., Nemes.L., (1999) Organisational Design: Dynamically Creating and Sustaining Integrated Virtual Enterprises, Proc IFAC World Congress, Han-Fu Chen, Dia-Zhan Cheng and Ji-Feng Zhang (Eds) Vol-A, London: Elsevier, pp189-194

C4ISR Working Group, (1998) Levels of Information Systems Interoperability, US DoD C4ISR Architecture Working Group, 30 March 1998

Doumeingts, G.,Vallespir, B., Chen, D., (1998) GRAI Grid Decisional Modeling, in Handbook on Architectures of Information Systems, Berlin: Springer Verlag, pp313-338

IFIP-IFAC Task Force (1999) Generalised Enterprise Reference Architecture and Methodology (GERAM) Version 1.6.3. Available at http://www.cit.gu.edu.au/ bernus/taskforce/geram/versions/

Olegario, C., (2001) A Partial Enterprise Model for the Management and Control in an Extended Enterprise Scenario, Masters Dissertation, School of Computing and Information Technology, Brisbane: Griffith University.

Tølle, M., Bernus,P., and Vesterager,J., (2002) Reference Models for Virtual Enterprises, Proceedings of PRO-VE'02, Sesimbra, May 2002

Vernadat, F. (1996) Enterprise Modelling and Integration: Principles and Applications, London : Chapman \& Hall.

Vesterager, J., Bernus P., Larsen L.B., Pedersen J.D. \& Tølle M. (2001) Use of GERAM as Basis for a Virtual Enterprise Framework Model", in "Global Engineering, Manufacturing and Enterprise Networks", ed. J. Mo and L. Nemes, Kluwer, pp.75-82.

Williams, T.J.,(1994) The Purdue Enterprise Reference Architecture, Computers in Industry, Vol 24, No 2-3, Special Issue on CIM, pp141-158.

Zhou, M., Nemes, L., Mo, J., Shinonome, M., Hasimoto, H., Fuse, A, Bernus, P., Uppington, G., (1998) Requirement Analysis for Virtual Manufacturing Enterprise, Proc. DIISM98, London: Chapman \& Hall.

Zwegers, A., Hannus M. \& Tølle M., Integration issues in virtual enterprises supported by an architectural framework, Proceedings of the IMS Forum 2001 